\title{
PRODUCCIÓN A LA INTEMPERIE DE UN CONSORCIO MICROBIANO FOTOSINTÉTICO FIJADOR DE NITRÓGENO EN CULTIVO POR LOTE Y SEMICONTINUO EN UN FOTOBIORREACTOR DE PUENTE AÉREO DE CARA PLANA
}

\author{
Dulce Jazmín HERNÁNDEZ MELCHOR ${ }^{1}$, Eliseo CRISTIANI URBINA ${ }^{2}$, Ronald FERRERA CERRATO ${ }^{3}$, \\ José Fernando ESPARZA GARCÍA ${ }^{1}$ y Rosa Olivia CAÑIZARES VILLANUEVA ${ }^{1 *}$
}

\footnotetext{
${ }^{1}$ Departamento de Biotecnología y Bioingeniería, Centro de Investigación y de Estudios Avanzados. Instituto Politécnico Nacional. Avenida Instituto Politécnico Nacional 2508, San Pedro Zacatenco, Ciudad de México, México, C. P. 07360

${ }^{2}$ Departamento de Ingeniería Bioquímica, Escuela Nacional de Ciencias Biológicas, Instituto Politécnico Nacional. Avenida Wilfrido Massieu, Unidad Profesional Adolfo López Mateos, Ciudad de México, México, C. P. 07738

${ }^{3}$ Instituto de Recursos Naturales, Colegio de Postgraduados, Campus Montecillos. Carretera México-Texcoco, kilómetro. 36.5, Montecillo, Texcoco, Estado de México, México, C. P. 56230

*Autor de correspondencia: rcanizar@cinvestav.mx
}

(Recibido julio 2015; aceptado noviembre 2016)

Palabras clave: cianobacteria, autotrofía, biofertilizante

\section{RESUMEN}

Las cianobacterias son organismos fotótrofos oxigénicos que tienen la capacidad de formar consorcios. Una estrategia que ha sido utilizada para mejorar la productividad de cianobacterias y microalgas en fotobiorreactores es producirlas en cultivo semicontinuo. En nuestro grupo de trabajo hemos estudiado un consorcio microbiano (CM) fotosintético con capacidad de fijar nitrógeno, constituido principalmente por cianobacterias, el cual tiene uso potencial como biofertilizante. El objetivo de este estudio fue evaluar la capacidad de producción de biomasa del CM en dos fotobiorreactores (FBR) de puente aéreo de cara plana $(50$ y $150 \mathrm{~L})$ a la intemperie, en cultivo por lote en ambos FBR y en cultivo semicontinuo en el FBR de mayor volumen. La máxima concentración de biomasa en cultivo por lote se obtuvo en el FBR de $150 \mathrm{~L}$, con un valor de $600 \mathrm{mg} / \mathrm{L}$, en comparación a la biomasa obtenida en el FBR de $50 \mathrm{~L}$, la cual fue igual a $385 \mathrm{mg} / \mathrm{L}$. La productividad de biomasa en los FBR de 50 y $150 \mathrm{~L}$ fue de 0.77 y $1.19 \mathrm{mg} / \mathrm{L} / \mathrm{h}$, respectivamente. El cultivo del CM en semicontinuo se realizó por tres ciclos ( 7,7 y 5 d cada uno, respectivamente). Se obtuvo un aumento de la productividad de biomasa tras cada ciclo, con valores de $1.86,1.94 \mathrm{y}$ $2.94 \mathrm{mg} / \mathrm{L} / \mathrm{h}$. Asimismo, los valores máximos de actividad de nitrogenasa se obtuvieron al final de cada ciclo, los cuales fueron de 4963,5366 y $5689 \mathrm{nmol}$ eteno/g peso seco/h, respectivamente. Estos valores fueron superiores a los obtenidos en cultivo por lote. En conclusión, es factible producir el CM en los FBR en cultivo por lote y semicontinuo a la intemperie, sin afectar su capacidad de fijación de nitrógeno.

Key words: cyanobacteria, autotrophy, biofertilizer 


\begin{abstract}
Cyanobacteria are oxygenic phototrophic organisms that have the capacity to form consortia. One strategy that has been used to improve the productivity of cyanobacteria and algae in photobioreactors is to produce them in semicontinuous cultures. In our research group, a photosynthetic microbial consortium (MC) with nitrogen fixing capacity was studied. It consists mainly of cyanobacteria and could potentially be used as a biofertilizer. The aim of this study was to evaluate the capability to produce biomass of MC in two flat-panel airlift photobioreactors (PBR) (50 y $150 \mathrm{~L})$ in batch culture, and in semicontinuous culture in a PBR with $150 \mathrm{~L}$ capacity, outdoors. The maximum biomass concentration in batch culture was obtained in the PBR of $150 \mathrm{~L}$, with a value of $600 \mathrm{mg} / \mathrm{L}$, in comparison with the biomass concentration obtained in the PBR of $50 \mathrm{~L}$, which was equal to $385 \mathrm{mg} / \mathrm{L}$. The biomass productivity at the end of the batch culture in both PBR $(50$ and $150 \mathrm{~L})$ was 0.77 and $1.19 \mathrm{mg} / \mathrm{L} / \mathrm{h}$, respectively. The semicontinuous culture of CM was operated during three cycles $(7,7$ and $5 \mathrm{~d}$, respectively), attaining an increase in the biomass productivity following each cycle, with values of $1.86,1.94$ and $2.94 \mathrm{mg} / \mathrm{L} / \mathrm{h}$, respectively. Similarly, the maximum values of the nitrogenase activity were obtained at the end of each cycle with values of 4963 , 5366 and $5689 \mathrm{nmol}$ ethene/g dry weight/h, respectively. These were higher than the values obtained in batch cultures. In conclusion, it was possible to produce the MC in the PBR in batch and semicontinuous mode outdoors, without affecting the nitrogen fixation capacity.
\end{abstract}

\section{INTRODUCCIÓN}

Las cianobacterias son organismos fotosintéticos con requerimientos simples para su crecimiento (luz, $\mathrm{CO}_{2}, \mathrm{~N}, \mathrm{P}, \mathrm{K}$ ), las podemos encontrar distribuidas en diversos ambientes acuáticos y terrestres (Thajuddin y Subramanian 2005). Tienen la capacidad de producir compuestos con alto valor agregado para diferentes áreas de interés biotecnológico como son alimentos, aditivos, pigmentos, dietas para acuacultura, metabolitos secundarios, o bien ser empleadas en el tratamiento de aguas residuales. También han sido ampliamente utilizadas por su capacidad de fijación de $\mathrm{CO}_{2}$, remoción de óxido nítrico y su capacidad de fijar nitrógeno (Carvalho et al. 2006). Esta última característica la poseen cianobacterias filamentosas que tienen la aptitud de diferenciar células denominadas heterocistos en las que se sintetiza y se lleva a cabo la función del complejo enzimático nitrogenasa (Singh y Sharma 2012). Por esta cualidad su biomasa es ampliamente utilizada como biofertilizante en países tropicales, de manera particular en cultivos de arroz (Goyal 2002). Sin embargo, a pesar de su enorme potencial, el número de aplicaciones que tienen las cianobacterias a escala industrial aún es limitado, por lo que es necesario desarrollar tecnologías de producción en fotobiorreactores a gran escala.

Una estrategia desarrollada para favorecer la producción de biomasa de cianobacterias a gran escala y que sea económicamente factible es operar los fotobiorreactores en régimen semicontinuo a la intemperie. Sin embargo, el ciclo de luz no puede ser controlado en esta condición, lo que afecta su productividad (Chen et al. 2011). Este tipo de sistema de cultivo (cultivo semicontinuo del tipo por lote secuencial o lote repetido) ofrece ventajas tales como obtener una proporción elevada y constante de inóculo y altas tasas de crecimiento. Además de que se prolonga la operación de los reactores y con ello se reducen los costos de operación (Reichert et al. 2006). Por lo anterior, diversos autores han evaluado diferentes diseños de fotobiorreactores para asegurar el uso eficiente de la luz a la intemperie en cultivo semicontinuo (Innok et al. 2009). Por ejemplo, los diseños para la producción de Tetraselmis subcordiformis en un fotobiorreactor de puente aéreo rectangular de $10.5 \mathrm{~L}$ (Zheng et al. 2011) y Haematococcus pluvialis en un fotobiorreactor de puente aéreo de cara plana de 17 L (Issarapayup et al. 2009). Asimismo, Travieso et al. (2001) utilizaron un sistema semicontinuo con diferentes tasas de dilución en un fotobiorreactor tubular helicoidal de 20 L para la producción de Spirulina platensis. Los autores mencionados reportaron aumentos en la productividad de biomasa de las microalgas utilizadas, a través de un cultivo semicontinuo comparado con un cultivo por lote.

Al existir pocos informes sobre el uso del cultivo semicontinuo a la intemperie para la producción de cianobacterias y de microalgas, se requiere llevar a cabo mayor cantidad de estudios científicos con la fi- 
nalidad de determinar las condiciones más adecuadas para la producción de biomasa microalgal, así como de otros productos de interés biotecnológico. Nuestro grupo de trabajo aisló un consorcio microbiano fotosintético con uso potencial como biofertilizante por su elevada capacidad para fijar nitrógeno. El siguiente paso será desarrollar tecnologías de producción en cultivo por lote y semicontinuo a la intemperie con fotobiorreactores de diferente capacidad.

El objetivo del presente estudio fue evaluar la producción y capacidad de fijación de nitrógeno de un consorcio microbiano fotosintético en cultivo por lote y semicontinuo en un fotobiorreactor de puente aéreo de cara plana a la intemperie.

\section{MATERIALES Y MÉTODOS}

Microorganismos. Se utilizó un consorcio microbiano (CM) fotosintético, aislado de un campo arrocero ubicado en la comunidad de Alpuyeca, Estado de Morelos, México (Reyna-Velarde et al. 2012). El $\mathrm{CM}$ se propagó en un medio mineral sin fuente de nitrógeno $\left(\mathrm{BG} 11_{0}\right)\left(0.023 \mathrm{~g} / \mathrm{L} \mathrm{NaCl}, 0.04 \mathrm{~g} / \mathrm{L} \mathrm{K}_{2} \mathrm{HPO}_{4}\right.$. $3 \mathrm{H}_{2} \mathrm{O}, 0.075 \mathrm{~g} / \mathrm{L} \mathrm{MgSO}_{4} .7 \mathrm{H}_{2} \mathrm{O}, 0.036 \mathrm{~g} / \mathrm{L} \mathrm{CaCl}_{2}$. $2 \mathrm{H}_{2} \mathrm{O}, 0.006 \mathrm{~g} / \mathrm{L}$ ácido cítrico, $0.006 \mathrm{~g} / \mathrm{L}$ citrato férrico, $0.001 \mathrm{~g} / \mathrm{L}$ EDTA, $0.02 \mathrm{~g} / \mathrm{L} \mathrm{Na}_{2} \mathrm{CO}_{3}, 2.86 \mu \mathrm{g} / \mathrm{L}$ $\mathrm{H}_{3} \mathrm{BO}_{4}, 1.81 \mu \mathrm{g} / \mathrm{L} \mathrm{MnCl}_{2} \cdot 4 \mathrm{H}_{2} \mathrm{O}, 0.222 \mu \mathrm{g} / \mathrm{L}$ $\mathrm{ZnSO}_{4} \cdot 7 \mathrm{H}_{2} \mathrm{O}, 0.391 \mu \mathrm{g} / \mathrm{LNa}_{2} \mathrm{MoO}_{4} \cdot 2 \mathrm{H}_{2} \mathrm{O}, 0.079 \mu \mathrm{g} / \mathrm{L}$ $\mathrm{CuSO}_{4} \cdot 5 \mathrm{H}_{2} \mathrm{O}, 0.0494 \mu \mathrm{g} / \mathrm{LCo}\left(\mathrm{NO}_{3}\right)_{2} \cdot 6 \mathrm{H}_{2} \mathrm{O}$ ) (Rippka et al. 1979). La identificación de los microorganismos procariontes que integran al $\mathrm{CM}$ se llevó a cabo en un estudio previo por el grupo de trabajo a partir de técnicas de biología molecular (secuenciando un fragmento del gen $16 \mathrm{~S}$ ADNr), además de la identificación morfológica de los organismos eucariontes que lo integran mediante comparación con claves taxonómicas. La muestra utilizada para la identificación del CM fue tomada al final del cultivo por lote realizado en un fotobiorreactor tipo columna de burbuja de $11 \mathrm{~L}$. A partir de este estudio se lograron identificar a 21 microorganismos diferentes que constituyen al $\mathrm{CM}$, pertenecientes a los géneros: Rhodobacter, Devosia, Pedomicrobium, Aquaspirillum, Methylibium, Nannocystis, Flavobacterium, Aphanizomenon, Leptolyngbya, Anabaena, Monoraphidium, Chlorella, Cyclotella, Melosira, Cocconeis, Achnanthidium, Navicula, Fragilaria y Nitzschia (Hernández et al. 2016).

Las condiciones de operación propuestas para cada uno de los FBR, así como su configuración fueron diseñadas con el propósito de favorecer el crecimiento y mantenimiento de cada uno de los componentes que integran al CM a la intemperie, sin afectar su capacidad de fijación de nitrógeno.

Fotobiorreactores de puente aéreo de cara plana. Para la producción en lote del CM se utilizaron dos fotobiorreactores (FBR) de puente aéreo de cara plana de diferente volumen de operación (50 y 150 L), pero con las mismas relaciones geométricas, a los cuales se les realizó previamente su caracterización hidrodinámica y de transferencia de masa (Hernández 2015).

Para disminuir los costos de operación, los FBR y el medio de cultivo no se esterilizaron, además de que se operaron a la intemperie, lo que implica un ahorro de energía lumínica. Los FBR se lavaron con extran (Merck Millipore), agua destilada e hipoclorito de sodio para su asepsia. El medio de cultivo se preparó con agua destilada. Una vez inoculados los FBR se sellaron de la parte superior con una tapa de acrílico transparente y silicón, dejando únicamente una salida de aire para evitar su presurización.

Cultivo por lote y semicontinuo. Los experimentos de producción del CM a la intemperie se realizaron durante los meses de mayo-junio de 2013, que coincide con la primavera, en la Unidad Experimental de Cultivo de Algas (UECA) del Centro de Investigación y de Estudios Avanzados del Instituto Politécnico Nacional (CINVESTAV) Unidad Zacatenco, Ciudad de México (19³0'33"N 9907'46"O). El promedio de temperatura ambiente (temperatura de bulbo seco) y luz registradas a lo largo de los experimentos fueron $27.9 \pm 4{ }^{\circ} \mathrm{C}$ (mínima $23.5 \pm 2{ }^{\circ} \mathrm{C}$, máxima $35 \pm 2{ }^{\circ} \mathrm{C}$ ) y $276 \mu \mathrm{mol}$ fotones $/ \mathrm{m}^{2} / \mathrm{s}$ (mínimo de $31 \mu \mathrm{mol}$ fotones $/ \mathrm{m}^{2} / \mathrm{s}$, máximo de $789 \mu \mathrm{mol}$ fotones $\left./ \mathrm{m}^{2} / \mathrm{s}\right)$, respectivamente. La presión barométrica registrada en la ciudad fue de 784.4 mb (Estación meteorológica de la Escuela Nacional Preparatoria 9).

El inóculo inicial utilizado para la propagación del CM en el FBR de $50 \mathrm{~L}$ se obtuvo en dos fotobiorreactores tipo columna de burbujas de $11 \mathrm{~L}$ a la intemperie. La productividad de biomasa después de $14 \mathrm{~d}$ de operación en cultivo por lote en este fotobiorreactor fue de $0.86 \mathrm{mg} / \mathrm{L} / \mathrm{h}$. Una vez culminada la cinética por lote en el FBR de $50 \mathrm{~L}$, la biomasa obtenida se utilizó como inóculo para el FBR de $150 \mathrm{~L}$.

Las cinéticas de producción en lote del CM se llevaron a cabo en los dos FBR a la intemperie utilizando el medio mineral BG11 0 (Rippka et al. 1979), un porcentaje de inóculo del $33 \%(\mathrm{v} / \mathrm{v})$ y un flujo de aire de $0.6 \mathrm{vvm}$. La duración del cultivo por lote en los FBR de 50 y $150 \mathrm{~L}$ fue de $14 \mathrm{~d}$.

Con base en los resultados obtenidos en el cultivo por lote a la intemperie donde el crecimiento del $\mathrm{CM}$ no fue afectado por las condiciones ambientales (luz y temperatura) y con la finalidad de aumentar la 
productividad de biomasa a gran escala, se llevó a cabo la producción del $\mathrm{CM}$ en cultivo semicontinuo únicamente en el FBR de mayor volumen $(150 \mathrm{~L})$.

El cultivo semicontinuo del CM en el FBR de $150 \mathrm{~L}$ inició con un cultivo por lote, del cual una vez alcanzada la concentración máxima de biomasa, se retiró un $50 \%$ de volumen del cultivo y se sustituyó por medio fresco $\left(\mathrm{BG} 11_{0}\right)$, procedimiento que se realizó dos veces más. La duración de cada ciclo de cultivo se determinó con base en la máxima concentración de biomasa obtenida en la fase logarítmica de crecimiento en cultivo por lote y fue igual a $600 \mathrm{mg} / \mathrm{L}$. Los ciclos de cultivo tuvieron una duración de 7, 7 y $5 \mathrm{~d}$ cada uno.

Técnicas analíticas. Las muestras de $100 \mathrm{~mL}$ de los FBR se tomaron por las mañanas. La temperatura dentro del FBR se midió con un termómetro de mercurio sumergido en la parte central del mismo en ambas zonas (ascendente y descendente). La cantidad de luz incidente en ambas caras de cada FBR se obtuvo con un radiómetro Quantitherm (Light Meter Thermometer) Hansatech Instruments LTD (QRT1) PE32 1JLUK. La temperatura y la luz se midieron tres veces diariamente (mañana, medio día y tarde).

Peso seco de biomasa. Se determinó por filtración de $15 \mathrm{~mL}$ de cultivo a través de membranas de celulosa de $5 \mu \mathrm{m}$ de diámetro de poro (Millipore ${ }^{\circledR}$ ) previamente puestas a peso constante y se secaron en horno a $70{ }^{\circ} \mathrm{C}$ durante $24 \mathrm{~h}$ (Tredici et al. 1991, Dash y Mishra 1999).

Velocidad específica de crecimiento. La velocidad específica máxima de crecimiento $(\mu)$, obtenida en la fase exponencial, se calculó de acuerdo con la siguiente ecuación (Issarapayup et al. 2009):

$\mu=\frac{\ln \left(N_{2}\right)-\ln \left(N_{1}\right)}{t_{2}-t_{1}}$

Donde $\mathrm{N}_{1}$ y $\mathrm{N}_{2}(\mathrm{mg} / \mathrm{L})$ corresponden a la concentración de biomasa seca a los tiempos $t_{1}$ y $t_{2}\left(\mathrm{~d}^{-1}\right)$.

Actividad de nitrogenasa (ensayo de reducción de acetileno). La actividad de nitrogenasa fue determinada por el método propuesto por Venkataraman (1981), modificando el tiempo de incubación. Se colocaron $10 \mathrm{~mL}$ de cultivo en botellas serológicas de $25 \mathrm{~mL}$ selladas herméticamente y se reemplazó $1 \mathrm{~mL}$ de aire del espacio vacío por $1 \mathrm{~mL}$ de acetileno. Las botellas se incubaron durante $5 \mathrm{~d}$ a $21 \pm 2^{\circ} \mathrm{C}$ con una intensidad luminosa de $80 \mu \mathrm{mol}$ fotones $/ \mathrm{m}^{2} / \mathrm{s}$ y un fotoperiodo (luz:oscuridad) de 12:12 h. Se evaluó la presencia de eteno con un cromatógrafo de gases Varian CP3380 FID bajo las siguientes condiciones:
$\mathrm{T}$ columna $=60{ }^{\circ} \mathrm{C}$, T inyector $=200{ }^{\circ} \mathrm{C}, \mathrm{T}$ detector $=250{ }^{\circ} \mathrm{C}$; columna: carbowax 1540 /porapak Q 1/8" $\times 6 \mathrm{ft}$; acarreador: $\mathrm{N}$ gas, 15 psi.

Contenido de clorofila $\boldsymbol{a}(\mathrm{Chl} \boldsymbol{a})$. Se utilizó la técnica de extracción con metanol al $90 \%$ descrita por Azov (Olvera et al. 2003) y se tomaron lecturas de absorbancia a 665 y $750 \mathrm{~nm}$ en un espectrofotómetro Genesys-10UV (Thermo Electron Corporation, USA).

Contenido de nitratos y amonio. La cuantificación de nitratos $\left(\mathrm{NO}_{3}{ }^{-}\right)$se llevó a cabo por el método de brucina-ácido sulfámico (APHA 1992) y la determinación de amonio $\left(\mathrm{NH}_{4}{ }^{+}\right)$por el método del fenato número132 C (APHA 1971).

pH. El valor de $\mathrm{pH}$ se midió en todas las muestras con un potenciómetro de laboratorio Orion 4129A calibrado previamente con soluciones de buffer a $\mathrm{pH}$ 4,7 y 9 a temperatura ambiente.

\section{RESULTADOS Y DISCUSIÓN}

\section{Cultivo por lote}

Se detectó un perfil similar de producción de biomasa del CM en base seca durante el cultivo por lote en los dos FBR (Fig. 1a). La fase de adaptación fue de aproximadamente $7 \mathrm{~d}$ y posteriormente inició la fase exponencial de crecimiento hasta alcanzar la concentración máxima de biomasa al día 14 del cultivo. Lo que probablemente indica la adaptación del cultivo a las condiciones hidrodinámicas dentro de los FBR (tiempo de mezclado, fracción de gas retenido, Reynolds, etc.; Hernández 2015), así como a la distribución de nutrientes y de luz. Asimismo, los resultados anteriores indican que al conservar la configuración geométrica de los FBR, es factible escalar el proceso a un mayor volumen sin afectar la producción de biomasa, resultado que se refleja en el aumento de la productividad de biomasa observado conforme se aumentó la capacidad del FBR (Cuadro I). Lo anterior sin verse afectado por las condiciones ambientales presentadas a la intemperie (luz y temperatura).

La temperatura promedio registrada en el interior de los FBR de 50 y $150 \mathrm{~L}$ operando en lote fue de $24 \pm 5^{\circ} \mathrm{C}$ y $23 \pm 4^{\circ} \mathrm{C}$, respectivamente y el promedio de luz incidente en cada FBR fue de 214 y $210 \mu \mathrm{mol}$ fotones $/ \mathrm{m}^{2} / \mathrm{s}$, respectivamente. Los parámetros de luz y de temperatura previamente mencionados, no afectaron la producción de biomasa del CM en lote, lo que probablemente indica una adaptación del CM a las condiciones de operación de los FBR, así como a las ambientales. 
a)

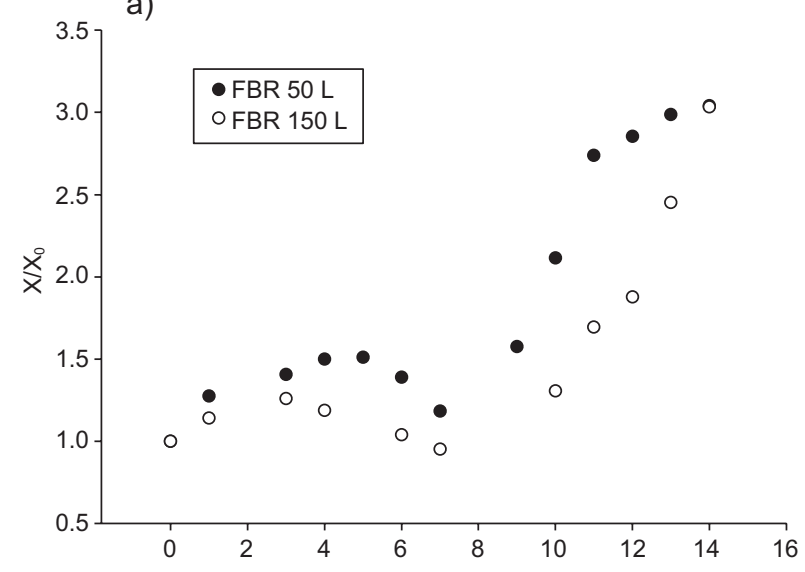

b)

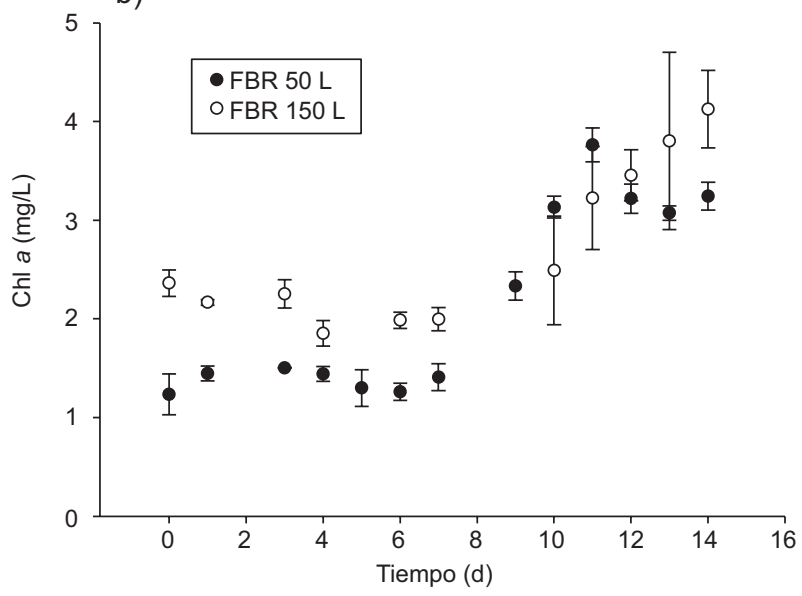

Fig. 1. (a) Relación de producción de biomasa respecto a la biomasa inicial $\left(\mathrm{X} / \mathrm{X}_{0}\right)$ y (b) concentración de clorofila $a(\mathrm{Chl} a)$ del consorcio microbiano fotosintético respecto al tiempo en los fotobiorreactores (FBR) de puente aéreo de cara plana de 50 y $150 \mathrm{~L}$ en cultivo por lote

La adaptación de cultivos de microalgas para su producción en FBR a la intemperie ha sido previamente reportada por Richmond y Cheng-Wu (2001), quienes llevaron a cabo la producción de Nannochloropsis sp. en FBR de cara plana de 200 L. También Slegers et al. (2011) estudiaron la producción de
Phaeodactylum tricornutum y Thalassiosira pseudonana en FBR de cara plana de diferente tamaño en diferentes regiones del mundo, lo que les permitió encontrar las condiciones óptimas de luz, temperatura y orientación de los los mismos para su producción.

A lo largo del cultivo por lote se cuantificó la variación del contenido de clorofila $a(\mathrm{Chl} a)$ del CM respecto al tiempo de incubación en los FBR (Fig. 1b). La medición de este parámetro es importante ya que se considera una medida indirecta del contenido de organismos fotosintéticos presentes en el cultivo (Whitton y Potts 2000). Durante la cinética en cultivo por lote se observó que la concentración de clorofila $a$ se mantuvo prácticamente constante los primeros 7 d de incubación en ambos FBR, lo que sugiere que se presentó una fase de adaptación de los organismos fotosintéticos presentes en el CM durante estos días (Fig. 1b). Posteriormente en el FBR de 150 L, el contenido de clorofila $a$ aumentó de manera exponencial hasta finalizar el experimento. En contraste, en el FBR de $50 \mathrm{~L}$ a partir del día 7 y hasta el día 11 del cultivo por lote, se observó un aumento de la concentración de Chl $a$ respecto al tiempo, para posteriormente disminuir del día 12 al 14. Estos tiempos coinciden con el cambio en el comportamiento mostrado para la producción de biomasa, lo que indica probablemente el inicio de la desaceleración del crecimiento microbiano (Fig. 1a). Los perfiles de clorofila $a$ descritos anteriormente probablemente se deban a la adaptación de cada microorganismo que conforma al CM a las diferentes escalas e hidrodinámica de los FBR (Hernández 2015), así como a las condiciones ambientales (luz y temperatura), ya que los experimentos se hicieron a la intemperie.

La importancia del CM utilizado en el presente trabajo radica en su capacidad de fijar nitrógeno, parámetro que determina su posible aplicación como biofertilizante. Por lo que fue necesario analizar su capacidad de fijación de nitrógeno en los dos FBR y determinar si el volumen de operación del FBR afecta esta capacidad. Los valores máximos de actividad de nitrogenasa obtenidos al final del cultivo por lote

CUADRO I. PRODUCTIVIDAD, VELOCIDAD ESPECÍFICA DE CRECIMIENTO, $\mathrm{pH}$, ACTIVIDAD DE NITROGENASA, LUZ INCIDENTE Y TEMPERATURA DEL CONSORCIO MICROBIANO FOTOSINTÉTICO EN LOS FOTOBIORREACTORES DE PUENTE AÉREO DE CARA PLANA DE 50 Y 150 L AL FINAL DEL CULTIVO POR LOTE

\begin{tabular}{lcccccc}
\hline FBR & $\begin{array}{c}\text { Productividad } \\
(\mathrm{mg} / \mathrm{L} / \mathrm{h})\end{array}$ & $\mu / \mathrm{h}$ & $\mathrm{pH}$ & $\begin{array}{c}\text { Actividad de nitrogenasa } \\
(\mathrm{nmol} \text { eteno/g peso seco/h })\end{array}$ & $\begin{array}{c}\text { Luz incidente } \\
\left(\mu \mathrm{mol} \text { fotones } / \mathrm{m}^{2} / \mathrm{s}\right)\end{array}$ & $\begin{array}{c}\text { Temperatura } \\
\text { interior }\left({ }^{\circ} \mathrm{C}\right)\end{array}$ \\
\hline $50 \mathrm{~L}$ & $0.77 \pm 0.0682$ & 0.005 & $7.32-9.61$ & $3093 \pm 113.1$ & 214 & $24 \pm 5$ \\
$150 \mathrm{~L}$ & $1.19 \pm 0.0510$ & 0.007 & $7.89-9.64$ & $4814 \pm 183.8$ & 210 & $23 \pm 4$ \\
\hline
\end{tabular}


en cada uno de los FBR a la intemperie se muestran en la cuadro I. La actividad máxima de nitrogenasa obtenida en el FBR de $150 \mathrm{~L}$ fue aproximadamente $56 \%$ superior a la alcanzada en el FBR de $50 \mathrm{~L}$. Asimismo, las actividades de nitrogenasa obtenidas en este trabajo con el CM fueron superiores a lo reportado en la literatura para Anabaena variabilis Sao (2678 nmol eteno/g peso seco/h) y Nostoc muscorum $\mathrm{DOH}$ (2237 nmol eteno/g peso seco/h; Kannaiyan et al. 1997, Kannaiyan 2002).

Existen diferentes parámetros que pueden afectar la actividad de nitrogenasa del cultivo, tal como la cantidad de $\mathrm{O}_{2}, \mathrm{~N}_{2}, \mathrm{NH}_{4}{ }^{+}$y $\mathrm{NO}_{3}{ }^{-}$en el medio, así como el pH y la temperatura (Brock et al. 2004). Por lo que en el presente trabajo se estudió la variación de la concentración de $\mathrm{NO}_{3}{ }^{-}$y $\mathrm{NH}_{4}{ }^{+}$en los dos $\mathrm{FBR}$ durante el cultivo por lote. Conocer la variación de estos dos nutrientes es importante debido a que a concentraciones elevadas $\left(1240 \mathrm{mg} / \mathrm{L} \mathrm{de} \mathrm{NO}_{3}{ }^{-}\right.$y $18 \mathrm{mg} / \mathrm{L} \mathrm{de} \mathrm{NH}_{4}{ }^{+}$) pueden inhibir el funcionamiento del complejo enzimático nitrogenasa, encargado de llevar a cabo la fijación biológica de nitrógeno (Almon y Boger 1988, Li et al. 2010). Las concentraciones más elevadas fueron 0.66 y $0.24 \mathrm{mg} / \mathrm{L}$ de nitratos y 3.58 y $3.23 \mathrm{mg} / \mathrm{L}$ de amonio en los FBR de 50 y $150 \mathrm{~L}$, respectivamente. Estas concentraciones son inferiores a las reportadas por Almon y Boger (1988) y Li et al. (2010) como inhibidoras del complejo nitrogenasa, por lo que se puede asegurar que la enzima encargada del proceso de fijación de nitrógeno no fue afectada por la presencia y concentración de estos dos compuestos en el cultivo.

También se llevó a cabo el seguimiento del $\mathrm{pH}$ a través del tiempo en los FBR. El pH del medio es un parámetro importante ya que cada uno de los organismos que conforman el CM funcionan de manera adecuada a diferente $\mathrm{pH}$. Además de que su variación a través del tiempo puede afectar parámetros como el funcionamiento de enzimas, crecimiento celular, producción de exopolisacáridos, etc. (Morales et al. 2002, Otero y Vicenzini 2004). El cuadro I muestra el intervalo de $\mathrm{pH}$ registrado en cada FBR en cultivo por lote. Estos valores permanecieron en el rango de 7-10 sin requerir ser controlados mediante la adición de sustancias amortiguadoras. Dicho rango resulta adecuado para el crecimiento de cada uno de los organismos presentes en el CM como proteobacterias, cianobacterias, flavobacterias, microalgas y diatomeas (Eroğlu et al. 2008, Roubeix y Lancelot 2008, Chaudhary et al. 2012, Hadas et al. 2013, Silaban et al. 2013, Hernández 2015).

Algunos de los parámetros previamente mencionados fueron estudiados por Tsyngankov et al.
(1997), quienes encontraron las condiciones que favorecían la actividad de Mo-nitrogenasa en Anabaena variabilis, las cuales fueron $\mathrm{pH}$ de 6.5 - 7, temperatura de $27-40{ }^{\circ} \mathrm{C}$ y una intensidad de luz de $421 \mu \mathrm{mol}$ fotones $/ \mathrm{m}^{2} / \mathrm{s}$ en un FBR de columna de burbujas de $500 \mathrm{~mL}$. Cianobacterias del género Anabaena forman parte del CM en estudio y son de las más abundantes. Aún cuando en esta investigación las condiciones de $\mathrm{pH}$, temperatura y luz en los dos FBR a la intemperie fueron diferentes a las reportadas por los autores anteriores, resultaron adecuadas para el crecimiento del CM en el tipo y escala de FBR estudiado, como lo demuestra el valor de fijación de nitrógeno. Nuestra experiencia indica que pudiera deberse a la interacción que existe entre los miembros del CM, así como al intercambio de metabolitos, lo que favorece la regulación y el crecimiento de cada organismo presente (Paerl y Pinckney 1996).

Los resultados anteriores muestran que el FBR de $150 \mathrm{~L}$ en cultivo por lote es el más adecuado para la producción de biomasa y mantenimiento de la actividad de nitrogenasa del CM.

\section{Cultivo semicontinuo}

Para mejorar la productividad de biomasa del CM a gran escala, se llevó a cabo una cinética de producción de biomasa en cultivo semicontinuo empleando el FBR de 150 L por tres ciclos. Se monitoreó la variación de la concentración de biomasa en base seca a lo largo del tiempo de incubación durante este régimen de cultivo (Fig. 2). El sistema inició con un cultivo por lote que operó durante $14 \mathrm{~d}$ hasta alcanzar una concentración de biomasa de $600 \mathrm{mg} / \mathrm{L}$ en base seca

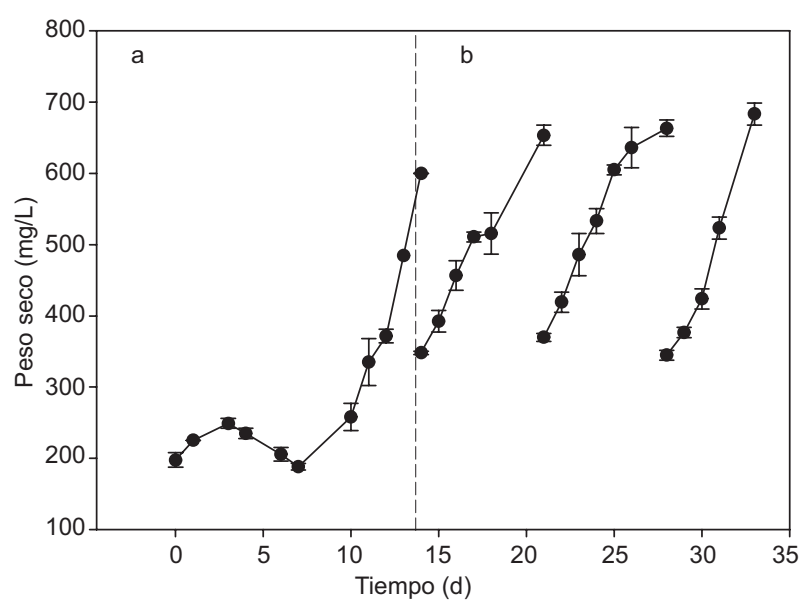

Fig. 2. Producción de biomasa del consorcio microbiano fotosintético en el fotobiorreactor de puente aéreo de cara plana de 150 L en cultivo: (a) por lote y (b) semicontinuo por tres ciclos 
CUADRO II. PRODUCTIVIDAD, VELOCIDAD ESPECÍFICA DE CRECIMIENTO, CLOROFILA $a$, pH, ACTIVIDAD DE NITROGENASA, LUZ INCIDENTE Y TEMPERATURA DEL CONSORCIO MICROBIANO FOTOSINTÉTICO EN EL FOTOBIORREACTOR DE PUENTE AÉREO DE CARA PLANA DE 150 L AL FINAL DE CADA CICLO EN CULTIVO SEMICONTINUO

\begin{tabular}{lccccccc}
\hline Ciclo & $\begin{array}{c}\text { Productividad } \\
(\mathrm{mg} / \mathrm{L} / \mathrm{h})\end{array}$ & $\mu / \mathrm{h}$ & $\begin{array}{c}\text { Clorofila } a \\
(\mathrm{mg} / \mathrm{L})\end{array}$ & $\mathrm{pH}$ & $\begin{array}{c}\text { Actividad de nitrogenasa } \\
(\mathrm{nmol} \text { eteno/ } \mathrm{g} \text { peso seco } / \mathrm{h})\end{array}$ & $\begin{array}{c}\text { Luz incidente } \\
\left(\mu \mathrm{mol} \text { fotones } / \mathrm{m}^{2} / \mathrm{s}\right)\end{array}$ & $\begin{array}{c}\text { Temperatura } \\
\text { interior }\left({ }^{\circ} \mathrm{C}\right)\end{array}$ \\
\hline 1 & $1.82 \pm 0.049$ & 0.0035 & $5.9 \pm 0.103$ & $7.7-9.5$ & $4963 \pm 10.89$ & 271 & $19 \pm 2$ \\
2 & $1.75 \pm 0.194$ & 0.0042 & $6.1 \pm 0.216$ & $7.9-9.2$ & $5366 \pm 11.53$ & 182 & $18 \pm 3$ \\
3 & $2.82 \pm 0.119$ & 0.0063 & $6.2 \pm 0.070$ & $7.6-10$ & $5689 \pm 15.85$ & 244 & $23 \pm 4$ \\
\hline
\end{tabular}

(Fig. 2a). Posteriormente, se cambió al modo de cultivo semicontinuo con un recambio del $50 \%$ (volumen del cultivo por medio fresco) para los tres ciclos (Fig. 2b). La productividad de biomasa en cada ciclo $\left(1^{\circ}, 2^{\circ}\right.$ y $\left.3^{\circ}\right)$ del cultivo semicontinuo fue de $1.82,1.75$ y $2.82 \mathrm{mg} / \mathrm{L} / \mathrm{h}$, respectivamente. Se observó un aumento en la velocidad específica de crecimiento tras cada ciclo de cultivo (Cuadro II). Un comportamiento similar fue reportado previamente por Fábregas et al. (1996), quienes concluyeron que algunas de las ventajas de trabajar en un sistema semicontinuo son mantener una cantidad de inóculo constante y una alta velocidad específica de crecimiento. Asimismo, Henrard et al. (2011) aumentaron la producción de biomasa y la velocidad específica de crecimiento de Cyanobium sp. operando en cultivo semicontinuo un FBR tubular vertical de $2 \mathrm{~L}$, con una tasa de recambio del $50 \%$ por 10 ciclos.

La productividad de biomasa del $\mathrm{CM}$ obtenida en cultivo semicontinuo en el FBR de $150 \mathrm{~L}$ a la intemperie fue aproximadamente dos veces superior a la del cultivo por lote con el mismo FBR. Este comportamiento ha sido reportado por diversos autores, quienes han estudiado la producción de microalgas en FBR de diferentes escalas y geometrías, operando en cultivo semicontinuo, en el que lograron mejorar la producción de biomasa comparada con el cultivo por lote (Fabregas et al. 1996, Travieso et al. 2001, Reichert et al. 2006, Sun et al. 2009).

La temperatura promedio registrada en el interior del FBR de $150 \mathrm{~L}$ durante el cultivo semicontinuo fue de $20 \pm 4^{\circ} \mathrm{C}$ y el promedio de luz incidente fue de $233 \mu \mathrm{mol}$ fotones $/ \mathrm{m}^{2} / \mathrm{s}$. Estos valores fueron similares a los obtenidos en cultivo por lote en el mismo FBR.

En el cuadro II se muestran los resultados de concentración de Chl $a$ obtenidos al final de cada ciclo de cultivo, los cuales fueron de 5.9, 6.1 y $6.2 \mathrm{mg} / \mathrm{L}$, respectivamente. Con cada cambio de ciclo se incrementó el contenido de $\mathrm{Chl} a$ aproximadamente un $5 \%$, lo que indica una adaptación de los organismos fotosintéticos que conforman al CM a la escala del FBR y a las condiciones de luz y temperatura registradas a lo largo del cultivo semicontinuo (19 d).

La variación de $\mathrm{pH}$ a lo largo del cultivo semicontinuo en los diferentes ciclos se presenta en la cuadro II. Se observa que los valores fueron similares a los obtenidos previamente en cultivo por lote en el mismo FBR (150 L) y que son adecuados para el crecimiento de los organismos que integran al CM.

Con la finalidad de detectar si los niveles de nitrato y amonio residuales podrían afectar la actividad de nitrogenasa en el sistema de cultivo semicontinuo, se determinó la concentración de estos compuestos a lo largo de los ciclos estudiados. Se encontró que los máximos valores registrados fueron $0.79 \mathrm{y}$ $3.2 \mathrm{mg} / \mathrm{L}$, respectivamente. Los cuales no son superiores a los mencionados por Almon y Boger (1988) y Li et al. (2010) como inhibitorios del funcionamiento de la enzima. Asimismo, se midió la variación de la actividad de nitrogenasa del CM en los tres ciclos y se encontró que los valores máximos al final de cada ciclo fueron 4963, 5366 y $5689 \mathrm{nmol}$ eteno/g peso seco/h (Cuadro II). Dichos valores fueron superiores a lo obtenido en el cultivo por lote en el mismo FBR y a lo reportado en la literatura (Kannaiyan et al. 1997, Kannaiyan 2002). Los resultados muestran que al operar el FBR de $150 \mathrm{~L}$ en cultivo semicontinuo se mejora la producción de biomasa sin afectar la fijación de nitrógeno del CM en estudio. Resultados semejantes fueron obtenidos por Moreno et al. (2003), quienes estudiaron la producción de Anabaena sp. ATCC 33047 en un estanque a la intemperie en un régimen semicontinuo. Estos autores demostraron que en el intervalo de temperatura de 21 a $34{ }^{\circ} \mathrm{C}$ en verano se favorece el crecimiento de la cianobacteria sin afectar el proceso de fijación de nitrógeno.

\section{CONCLUSIONES}

El cultivo semicontinuo favorece la producción de biomasa $(600 \mathrm{mg} / \mathrm{L})$ y la fijación de nitrógeno 
(4963-5689 $\mathrm{nmol}$ eteno/g peso seco/h) del CM en un FBR de $150 \mathrm{~L}$ operando a la intemperie, lo que asegura su producción constante y su posible empleo como biofertilizante.

Los resultados sugieren que el sistema semicontinuo podría ser utilizado como una estrategia prometedora para producir el CM a nivel comercial.

\section{AGRADECIMIENTOS}

Al Centro de Investigación y de Estudios Avanzados del Instituto Politécnico Nacional de México, por el financiamiento recibido para esta investigación. Al Consejo Nacional de Ciencia y Tecnología (CONACyT) de México, por la beca otorgada para llevar a cabo los estudios doctorales de la primera autora.

\section{REFERENCIAS}

Almon H. y Boger P. (1988). Nitrogen and Hydrogen Metabolism: Induction and Measurement. En: Methods in enzymology. (L. Packer, A.N. Glazer, Eds.). Academic Press, INC. San Diego, California, pp.459-467.

DOI: $10.1016 / 0076-6879(88) 67050-9$

APHA (1971). Standard methods for the examination of water and wastewater, $13^{\text {th }}$ Edition. American Public Health Association, American Water Works Association, Water Pollution Control Federation, Washington, EUA, 1325 pp.

APHA (1992). Standard methods for the examination of water and wastewater, $13^{\text {th }}$ Edition. American Public Health Association, American Water Works Association, Water Pollution Control Federation, Washington, EUA, 1325 pp.

Brock J. T., Madigan M. T., Martinko J. M. y Parker J. (2004). Biología de los microorganismos. $10^{a}$ Edición. Pearson Education S.A., Madrid, España, 1089 pp.

Carvalho A. P., Meireles L. A. y Malcata F. X. (2006). Microalgal reactors: a review of enclosed system designs and performances. Biotechnol. Progr. 22, 1490-1506. DOI: $10.1021 / \mathrm{bp} 060065 \mathrm{r}$

Chaudhary V., Prasanna R. y Bhatnagar A. K. (2012). Influence of phosphorus and $\mathrm{pH}$ on the fungicidal potential of Anabaena strains. J. Basic. Microbiol. 53, 201-213. DOI: 10.1002/jobm.201100520

Chen C. Y., Yeh K. L., Aisyah R., Lee D. J. y Chang J. S. (2011). Cultivation, photobioreactor design and harvesting of microalgae for biodiesel production: A critical review. Bioresource Technol. 102, 71-81.

DOI: 10.1016/j.biortech.2010.06.159
Dash K. A. y Mishra P. C. (1999). Growth response of the blue-green alga, Westiellopsis prolifica in sewage enriched paper mill waste water. Rev. Int. Contam. Ambie. 15, 79-83.

Eroğlu I., Tabanoğlu A., Gündüz U., Eroğlu E. y Yücel M. (2008). Hydrogen production by Rhodobacter sphaeroides O.U. 001 in a flat plate solar bioreactor. Int. J. Hydrogen Energ. 32, 531-541.

DOI: 10.1016/j.ijhydene.2007.09.025

Fábregas J., Patiño M., Morales E. D., Domínguez A. y Otero A. (1996). Distinctive control of metabolic pathways by Chlorella autotrophica in semicontinuous culture. Can. J. Microbiol. 42, 1087-1090.

DOI: $10.1139 / \mathrm{m} 96-139$

Goyal S. K. (2002). A profile on algal biofertilizer. En: Biotechnology of Biofertilizers (S. Kannaiyan, Ed.). Narosa Publishing House, Nueva Delhi, India, pp.250-258.

Hadas O., Pinkas R., Malinsky-Rushansky N., ShalevAlon G., Delphine E., Berner T., Sukenik A. y Kaplan A. (2013). Physiological variables determined under laboratory conditions may explain the bloom of Aphanizomenon ovalisporum in Lake Kinneret. Eur. J. Phycol. 37, 259-267. DOI: 10.1017/S0967026202003645

Hernández M. D. J. (2015). Optimización de las condiciones de cultivo de un consorcio microbiano fotosintético de uso potencial como biofertilizante. Tesis de Doctorado. Departamento de Biotecnología y Bioingeniería. Centro de Investigación y de Estudios Avanzados del Instituto Politécnico Nacional, Ciudad de México, México, 154 pp.

Hernández M. D. J., Carmona J. J., Hidalgo L. M. E, Dendooven L., Marsch M. R. y Cañizares V. R. O. (2016). Identificación morfológica y filogenética de un consorcio microbiano fotosintético de posible interés biotecnológico. Hidrobiológica. 26, 311-321.

Henrard A. A., de Morais M. G. y Costa J. A. V. (2011). Vertical tubular photobioreactor for semicontinuous culture of Cyanobium sp. Bioresource Technol. 102, 4897-4900. DOI: 10.1016/j.biortech.2010.12.011

Innok S., Chunleuchanon S., Boonkerd N. y Teaumroong N. (2009). Cyanobacterial akinete induction and its application as biofertilizer for rice cultivation. J. Appl. Phycol. 21,737-744. DOI: 10.1007/s10811-009-9409-x

Issarapayup K., Powtongsookb S. y Pavasant P. (2009). Flat panel airlift photobioreactors for cultivation of vegetative cells of microalga Haematococcus pluvialis. J. Biotechnol. 142, 227-232.

DOI: 10.1016/j.jbiotec.2009.04.014

Kannaiyan S., Aruna S. J., Merina P. K. S. y Hall D. O. (1997). Immobilized cyanobacteria as a biofertilizer for rice crops. J. Appl. Phycol. 9, 167-174.

DOI: 10.1023/A:1007962025662 
Kannaiyan S. (2002). Biofertilizers for sustainable crop production. En: Biotechnology of Biofertilizers (S. Kannaiyan, Ed.). Narosa Publishing House, New Delhi, India, pp. 9-49.

Li Z., Yu J., Kyoung-Rae K. y Brand J. (2010). Nitrogen fixation by a marine non-heterocystous cyanobacterium requires a heterotrophic bacterial consort. Environ. Microbiol. 12, 1185-1193.

DOI: $10.1111 / \mathrm{j} .1462-2920.2010 .02159 . x$

Morales E., Rodríguez M., García D., Loreto C. y Marco E. (2002) Crecimiento, producción de pigmentos y exopolisacáridos de la cianobacteria Anabaena sp. PCC7120 en función del pH y $\mathrm{CO}_{2}$. Interciencia 27, 373-378.

Moreno J., Vargas M. A., Rodríguez H., Rivas J. y Guerrero M. G. (2003). Outdoor cultivation of a nitrogen-fixing marine cyanobacterium, Anabaena sp. ATCC 33047. Biomolecular Eng. 20, 191-197.

DOI: $10.1016 / \mathrm{S} 1389-0344(03) 00051-0$

Olvera R., Ríos E. y Vicente V. (2003). Manual de técnicas para el cultivo y extracción de Bioproductos a partir de Microalgas. Escuela Nacional de Ciencias Biológicas (ENCB), Instituto Politécnico Nacional (IPN), Ciudad de México, México, 70 pp.

Otero A. y Vincenzini M. (2004). Nostoc (cyanophyceae) goes nude: extracellular polysaccharides serve as a sink for reducing power under unbalanced $\mathrm{C} / \mathrm{N}$ metabolism. J. Phycol. 40, 74-81.

DOI: $10.1111 / \mathrm{j} .0022-3646.2003 .03-067 . x$

Paerl H. W. y Pinckney J. L. (1996). A mini-review of microbial consortia: their roles in aquatic production and biogeochemical cycling. Microbial. Ecol. 31, 225247. DOI: 10.1007/BF00171569

Reichert C. C., Reinehr C. O. y Costa J. A. V. (2006). Semicontinuous cultivation of the cyanobacterium Spirulina platensis in a closed photobioreactor. Braz. J. Chem. Eng. 23, 23-28. DOI: $10.1590 / \mathrm{S} 0104-66322006000100003$

Reyna-Velarde R., Hernández-Melchor D., VázquezHernández M. y Cañizares-Villanueva R. O. (2012). Isolation of a photosynthetic microbial consortium formed mainly by cyanobacteria with capacity to fix nitrogen. Int. J. Environ. Eng. 4, 183-196.

DOI: 10.1504/IJEE.2012.050793

Richmond A. y Cheng-Wu Z. (2001). Optimization of a flat plate glass reactor for mass production of Nannochloropsis sp. outdoors. J. Biotechnol. 85, 259-269. DOI: 10.1016/S0168-1656(00)00353-9

Rippka R., Deruelles J., Waterbury J. B., Herdman M. y Stanier R. Y. (1979). Generic assignments, strain histories and properties of pure cultures of cyanobacteria. J. Gen. Microbiol. 111, 1-61. DOI: 10.1099/00221287-111-1-1

Roubeix V. y Lancelot C. (2008). Effect of salinity on growth, cell size and silicification of an euryhaline freshwater diatom: Cyclotella meneghiniana Kütz. Transit. Waters Bull. 1, 31-38.

DOI: $10.1285 / \mathrm{i} 1825229 X v 2 n 1 \mathrm{p} 31$

Silaban A., Bai R., Gutiérrez-Wing M. T., Negulescu I. I. y Rusch K. A. (2013). Effect of organic carbon, C:N ratio and light on the growth and lipid productivity of microalgae/cyanobacteria coculture. Eng. Life Sci. 14, 47-56. DOI: 10.1002/elsc.201200219

Singh R. N. y Sharma S. (2012). Development of suitable photobioreactor for algae production - A review. Renew. Sust. Energ. Rev. 16, 2347-2353.

DOI: $10.1016 /$ j.rser.2012.01.026

Slegers P. M., Wijffels R. H., van Straten G. y van Boxtel A. J. B. (2011). Design scenarios for flat panel photobioreactors. Appl. Energ. 88, 3342-3353.

DOI: 10.1016/j.apenergy.2010.12.037

Sun L., Zhang S., Wang C. y Shi L. (2009). Effects of renewal regime on consumption of nitrogen and phosphorus, biomass and polysaccharide production by Porphyridium cruentum in semicontinuous culture. Memorias. 3rd International Conference on Bioinformatics and Biomedical Engineering (ICBBE). Beijing, China, 11-13 junio.

Thajuddin N. y Subramanian G. (2005). Cyanobacterial biodiversity and potential applications in biotechnology. Curr. Sci. 89, 47-57.

Travieso L., Hall D. O., Rao K. K., Benítez F., Sánchez E. y Borja R. (2001). A helical tubular photobioreactor producing Spirulina in a semicontinuous mode. Int. Biodeter. Biodegr. 47, 151-155. DOI: 10.1016/S0964-8305(01)00043-9

Tredici M. R., Carlozzi P., Chini-Zitelli G. y Materassi R. (1991). A vertical alveolar panel (VAP) for outdoor mass cultivation of microalgae and cyanobacteria. Bioresource Technol. 38, 153-159.

DOI: 10.1016/0960-8524(91)90147-C

Tsygankov A. A., Serebryakova L. T., Sveshnikov D. A., Rao K. K., Gogotov I. N. y Hall D. O. (1997). Hydrogen photoproduction by three different nitrogenases in whole cells of Anabaena variabilis and the dependence on pH. Int. J. Hydrogen Energy 22, 859-867.

DOI: 10.1016/S0360-3199(96)00242-X

Venkataraman G. S. (1981). Blue-green algae for rice production. Food and Agriculture Organization of the United Nations. Rome, Italy. 102 pp.

Whitton B. A. y Potts M. (2000). The ecology of cyanobacteria. Kluwer Academic Publishers. Dordrecht, Netherlands, 632 pp.

Zheng Y., Chen Z., Lu H. y Zhang W. (2011). Optimization of carbon dioxide fixation and starch accumulation by Tetraselmis subcordiformis in a rectangular airlift photobioreactor. Afr. J. Biotechnol. 10, 1888-1901. DOI: $10.5897 / \mathrm{AJB} 10.1620$ 